\title{
Télescope
}

Revue d'analyse comparée en administration publique

\section{La gouvernance multiniveau et la nouvelle gestion publique : des idées nouvelles à intégrer aux approches traditionnelles du fédéralisme dans le contexte de l'étude des relations intergouvernementales}

\section{Lisa Turkewitsch et Michael Stein}

Volume 19, numéro 1, hiver 2013

La gouvernance multiniveau

URI : https://id.erudit.org/iderudit/1017153ar

DOI : https://doi.org/10.7202/1017153ar

Aller au sommaire du numéro

Éditeur(s)

L'Observatoire de l'administration publique

ISSN

1929-3348 (numérique)

Découvrir la revue

Citer cet article

Turkewitsch, L. \& Stein, M. (2013). La gouvernance multiniveau et la nouvelle gestion publique : des idées nouvelles à intégrer aux approches traditionnelles du fédéralisme dans le contexte de l'étude des relations

intergouvernementales. Télescope, 19(1), 85-114.

https://doi.org/10.7202/1017153ar
Résumé de l'article

Le concept de gouvernance multiniveau peut aider àreconnaître et à comprendre de nouvelles tendances dansles relations intergouvernementales et l'élaboration depolitiques. Dans cet article, nous nous penchons sur les liens quiexistent entre la littérature sur l'administrationpublique, particulièrement celle portant sur la nouvelle gestionpublique, et la gouvernance multiniveau. Ces deux approches sontsusceptibles d'améliorer les approches traditionnellesutilisées pour comprendre le fonctionnement interne desrelations intergouvernementales dans les régimesfédéraux. Nous fournissons des exemples tirés detrois fédérations : l'Australie, le Canada etles États-Unis. Nous nous concentrons sur la façon dontla gouvernance multiniveau peut mettre en lumière leschangements dans les relations intergouvernementales (entre leséchelons supranational,

fédéral,étatique/provincial et municipal) ainsi quel'élargissement horizontal de la gouvernance dans cesmêmes régimes à des organisations nongouvernementales et à d'autres acteurs. 


\title{
LA GOUVERNANCE MULTINIVEAU ET LA NOUVELLE GESTION PUBLIQUE : DES IDÉES NOUVELLES À INTÉGRER AUX APPROCHES TRADITIONNELLES DU FÉDÉRALISME DANS LE CONTEXTE DE L'ÉTUDE DES RELATIONS INTERGOUVERNEMENTALES
}

\author{
Par Lisa Turkewitsch 1, Candidate au doctorat, Département des sciences politiques, \\ Université de Toronto • lisa.turkewitsch@utoronto.ca \\ Et Michael Stein, Professeur invité, Département des sciences politiques, Université de \\ Toronto•michael.stein@utoronto.ca \\ Traduit de l'anglais
}

\begin{abstract}
RÉSUMÉ Le concept de gouvernance multiniveau peut aider à reconnaître et à comprendre de nouvelles tendances dans les relations intergouvernementales et l'élaboration de politiques. Dans cet article, nous nous penchons sur les liens qui existent entre la littérature sur l'administration publique, particulièrement celle portant sur la nouvelle gestion publique, et la gouvernance multiniveau. Ces deux approches sont susceptibles d'améliorer les approches traditionnelles utilisées pour comprendre le fonctionnement interne des relations intergouvernementales dans les régimes fédéraux. Nous fournissons des exemples tirés de trois fédérations : l'Australie, le Canada et les États-Unis. Nous nous concentrons sur la façon dont la gouvernance multiniveau peut mettre en lumière les changements dans les relations intergouvernementales (entre les échelons supranational, fédéral, étatique/provincial et municipal) ainsi que l'élargissement horizontal de la gouvernance dans ces mêmes régimes à des organisations non gouvernementales et à d'autres acteurs.
\end{abstract}

\begin{abstract}
We argue that the concept of multi-level governance (MLG) can help to uncover and understand changing trends in intergovernmental relations and policy-making. We also consider the linkages between the public administration literature, particularly the new public management (NPM) approach, and the concept of multi-level governance. Both the MLG and NPM approaches can help to enhance traditional approaches to understanding internal intergovernmental relations in federal systems. We draw on empirical examples from three Western federal systems: Canada, the United States, and Australia. In particular, we focus on how the concept of MLG can help to illuminate changing forms of intergovernmental relations (supranational-federal-state/provincial-local) and as well as the horizontal expansion of governance in these systems to non-governmental organizations and other actors.
\end{abstract}

Pour citer cet article : Turkewitsch, L. et M. Stein (2013). « La gouvernance multiniveau et la nouvelle gestion publique : des idées nouvelles à intégrer aux approches traditionnelles du fédéralisme dans le contexte de l'étude des relations intergouvernementales », Télescope, vol. 19, n 1, p. 85-114.

Nous tenons à remercier Sara Amini, Bianca Salazar et Hyun Kyung (Christine) Lee pour leur aide précieuse à la recherche. Nous remercions également le lecteur anonyme pour ses suggestions et commentaires pertinents. 
$\mathrm{L}$ es spécialistes de l'administration publique ont de façon générale étudié la gouvernance collaborative et en réseau, les partenariats public-privé (PPP) et autres phénomènes du genre, menant ainsi à des avancées conceptuelles et théoriques dans ce domaine. Ces mêmes spécialistes ont mis en pratique le concept de la gouvernance multiniveau (GMN), un concept issu de travaux de l'Union européenne. Comme ils ont conduit leurs recherches sur ces bases depuis longtemps, ils peuvent s'interroger sur ce qu'il y a de nouveau dans le domaine de la GMN. D’un autre côté, plusieurs spécialistes du fédéralisme et des relations intergouvernementales, particulièrement ceux à l'extérieur de l'Europe, ne connaissent pas ce concept ou ont eu tendance à ne pas considérer son utilité dans le cadre de leurs recherches.

Nous avançons que le concept de la GMN peut aider à reconnaître et à comprendre les nouvelles tendances qu'on observe dans la gouvernance. Mettre ce concept en œuvre pourrait encourager les spécialistes du fédéralisme et des relations intergouvernementales à voir plus loin que la littérature traditionnelle et à prendre en considération de nouvelles recherches solides, à la fois théoriques et pratiques, réalisées par des spécialistes de l'administration publique, des géographes, des étudiants en études environnementales, en études autochtones et autres domaines connexes. Tandis que certains chercheurs utilisent explicitement l'expression " gouvernance multiniveau ", d'autres ont recours à des concepts très proches, dont la gouvernance en réseau et collaborative et les partenariats. Plusieurs de ces chercheurs, y compris ceux dont les travaux se concentrent sur les régimes fédéraux, n'adoptent pas les concepts traditionnels du fédéralisme, et nous pensons qu'il peut en être ainsi parce que ces concepts sont trop contraignants et risquent de ne pas cadrer avec leurs recherches empiriques. Pourtant, le fédéralisme peut être partie intégrante d'autres domaines d'études et ajouter de la profondeur aux recherches. Nous croyons que le concept de la GMN peut servir de terrain commun qui fera le pont entre les connaissances en matière de fédéralisme et d'autres domaines en englobant à la fois l'élargissement horizontal de la gouvernance et les ordres verticaux de gouvernement dans un régime fédéral ou unitaire décentralisé. L'approche GMN permet une interprétation plus large du fédéralisme (Stein et Turkewitsch, 2010b), ainsi que l'intégration de concepts relatifs à la littérature portant sur l'administration publique.

Notre article est construit autour de trois grandes sections. La première, de nature théorique, définit le concept de GMN et ses liens avec la littérature sur le fédéralisme de même que le concept de NGP et la littérature récente dans le domaine de l'administration publique. La deuxième section se penche sur l'évolution des formes de gouvernance dans trois régimes fédéraux occidentaux : l'Australie, le Canada et les États-Unis. Nous nous concentrons sur la façon dont ces deux concepts peuvent mettre en lumière l'évolution des relations intergouvernementales (gouvernements supranationaux, fédéraux, étatiques ou provinciaux et municipaux), ainsi que l'expansion horizontale de la gouvernance à des organisations non gouvernementales et à d'autres acteurs dans ces systèmes, souvent omise par les approches traditionnelles du fédéralisme. Plus particulièrement, nous nous attardons à la GMN de type II (Hooghe et Marks, 2003) et à sa pertinence dans 
l'analyse des changements qui s'opèrent dans les relations intergouvernementales et l'administration publique. Enfin, la dernière section présente une analyse comparative et une discussion conceptuelle sur la façon dont la GMN et les idées qui lui sont liées émanant de l'approche de la NGP et de la littérature sur l'administration publique peuvent ajouter aux travaux sur le fédéralisme en se basant sur une définition élargie des relations intergouvernementales.

\section{- LA GOUVERNANCE MULTINIVEAU, LE FÉDÉRALISME ET L'ADMINISTRATION PUBLIQUE}

\section{Définition de la gouvernance multiniveau}

Il n'existe pas de définition unique et communément acceptée de la gouvernance multiniveau. Pour la définir, nous intégrons donc certains des aspects les plus largement acceptés du concept de la gouvernance multiniveau ${ }^{2}$. Dans la recherche de Hooghe et Marks (2003) sur les types I et II de la GMN (dont nous parlerons plus loin), nous avons observé que la GMN peut désigner les modèles de plus en plus complexes d'élaboration de politiques, de prise de décision autoritaire et des relations intergouvernementales dans les régimes fédéraux et unitaires décentralisés. Ce concept élargi englobe le processus de prise de décision dans sa définition et sa portée les plus larges, l'augmentation de décideurs en nombre et en type (y compris des acteurs du secteur privé tels que les entreprises et les syndicats, les organisations non gouvernementales, des membres de mouvements sociaux et la société civile), ainsi que les couches multiples du processus de prise de décision. La GMN peut être vue comme une construction empirique substantive, un idéal normatif, et comme une approche conceptuelle (Stein et Turkewitsch, 2010b). Il est important de différencier ces usages, même s'il peut parfois être difficile de les séparer. Dans cet article, nous nous concentrerons principalement sur l'évaluation et la mise en œuvre de la GMN en tant qu'approche analytique, même si nous traiterons brièvement de la GMN en tant que construction substantive.

Hooghe et Marks (2003) font une distinction entre deux types de gouvernance multiniveau, soit le type I et le type II. La GMN de type I partage de nombreux points de ressemblance avec le fédéralisme. Selon Hooghe et Marks, elle désigne la gouvernance des domaines de compétence ayant trait aux affaires courantes et faisant appel à un nombre restreint d'ordres de gouvernement, lesquels peuvent être supranationaux, nationaux, régionaux, infranationaux ou locaux. Ces domaines de compétence " regroupent de multiples fonctions, dont nombre de responsabilités politiques $^{3}$ " (Hooghe et Marks, 2003, p. 236). De plus, " les frontières délimitant l'appartenance à ces domaines de compétence ne se coupent pas. Il en est ainsi pour les domaines de compétence d'un ordre de gouvernement donné et pour ceux de tous les ordres de gouvernement " (Hooghe et Marks, 2003, p. 236). Toutefois,

2 Dans une de nos recherches précédentes, nous avons examiné et analysé en détail les nombreuses définitions concurrentes de la gouvernance multiniveau (Stein et Turkewitsch, 2008a).

3 Toutes les citations sont des traductions libres. 
ces auteurs avancent que " même si les frontières délimitant l'appartenance aux domaines de compétence de la GMN de type I ne se coupent pas, les compétences, elles, sont souvent partagées ou se chevauchent. Par exemple, il y a eu une tendance à s'éloigner de la compartimentation associée aux régimes politiques fédéraux " (Hooghe et Marks, 2003, p. 236). Hooghe et Marks décrivent la GMN de type II comme une forme de gouvernance dans laquelle le nombre de compétences est plus vaste que limité, et dans laquelle les compétences ne relèvent pas d'un nombre restreint d'ordres gouvernementaux, mais s'étendent à plusieurs sphères territoriales, où les compétences sont spécifiques et non générales et où les domaines de compétence sont pensés pour être flexibles plutôt que durables (Hooghe et Marks, 2003, p. 237). Nous considérons que la GMN de type I désigne les interactions à l'intérieur des ordres verticaux de gouvernement (y compris les ordres municipal, régional et supranational qui sont souvent laissés de côté par les approches traditionnelles du fédéralisme), et nous considérons la GMN de type II comme représentative de l'élargissement horizontal ou externe du gouvernement vers les organisations non gouvernementales et d'autres acteurs.

En règle générale, ces deux processus coexistent. Hooghe et Marks (2003, p. 238) ont noté que la gouvernance de type II est généralement intégrée dans la gouvernance de type I. Nous avançons que le chevauchement entre les deux types fournit un terrain commun aux chercheurs en relations intergouvernementales et en administration publique, ainsi qu'aux spécialistes de domaines tels que les politiques environnementales et de santé. La question de savoir de quelle façon les processus de la GMN de type II sont intégrés dans les régimes de type I (les régimes fédéraux ou unitaires décentralisés) est aussi importante pour de futures recherches empiriques et l'enrichissement du concept.

La distinction faite entre ces types de GMN a été généralement acceptée et appliquée dans la littérature. Par contre, on pourrait encore les enrichir sur le plan théorique. Un débat a également cours sur la question de savoir si, lorsque considérés comme des constructifs substantifs, les types I et II de GMN se chevauchent effectivement ou non. Comme l'écrivait Piattoni (2010, p. 25), "Bache (2008) et Conzelman et Smith (2007) considèrent les deux types de gouvernance d'une façon plutôt simple, soit comme différentes façons d'organiser la prestation de services donnés dans les démocraties modernes. Dans ce sens, les compétences de type II peuvent également être territoriales et superposées aux compétences de type I. Des exemples assez communs de cette conception sont les centres nationaux de soins de santé, les districts scolaires, les consortiums industriels, etc. " D'autres chercheurs remettent en question le besoin de faire une distinction entre les types I et II de GMN (par exemple Blatter, 2004, cité dans Leo et Enns, 2009). Ces derniers font remarquer que dans de nombreuses études sur le contexte canadien, on ne fait nulle part état de dichotomies simples entre les types I et II de GMN (Leo et Enns, 2009, p. 94; Young et Leuprecht, 2004). Nous estimons qu'il est utile sur une base théorique de tenir compte des types I et II dans notre analyse des régimes fédéraux, même si notre article porte principalement sur les exemples empiriques de GMN de type II. Comme nous tenons principalement compte de la relation entre la littérature sur l'administration publique et la GMN, nous mettons 
l'accent sur la GMN de type II en raison de ses liens plus étroits avec le concept de la NGP que la GMN de type I avec la littérature sur l'administration publique. De plus, les exemples empiriques de la GMN de type II illustrent mieux les processus émergents de GMN dans les trois régimes fédéraux étudiés.

Les théoriciens de l'administration publique et des politiques publiques ont fait avancer la GMN par leurs contributions théoriques, même si celles-ci sont plutôt indirectes. De plus, ils utilisent différentes appellations théoriques pour décrire le nouveau phénomène de gouvernance qu'ils mettent en lumière, comme la " gouvernance en réseau ", la " gouvernance en comité " ou les " nouveaux modes de gouvernance ». Un des théoriciens les plus en vue en la matière est Donald Kettl (2000 et 2009). Selon Kettl :

À tous les échelons du gouvernement [aux États-Unis], les partenariats avec des acteurs à la fois gouvernementaux et non gouvernementaux ont proliféré à un rythme grandissant [ces dernières années]. Cet état de fait a rendu le gouvernement à la fois horizontal - dans sa recherche de coordination et d'intégration de services avec des partenaires non gouvernementaux fournisseurs de services - et vertical - à travers ses bureaucraties traditionnelle et hiérarchique et le fédéralisme multiniveau. Ce n'est pas tant que les relations horizontales ont supplanté les relations verticales; c'est plutôt que les liens horizontaux ont été ajoutés aux liens verticaux. Cela, en effet, était le précepte implicite du mouvement « réinventons notre gouvernement » des années 1990. (Kettl, 2000, p. 494)

Selon nous, la description de Kettl des relations multicouches horizontales et verticales a des similitudes avec l'affirmation de Hooghe et Marks (2003) selon laquelle les processus de la GMN de type II sont intégrés à la GMN de type I. Le choix du terme " intégré " suggère l'existence de niveaux de gouvernance de type I, suivis d'un élargissement vers une GMN de type II. Dans le même esprit, la description fournie par Kettl de l'élargissement des processus horizontaux "superposés " aux niveaux verticaux existants exemplifie le fait que l'élargissement horizontal de la gouvernance (ou GMN de type II) est un phénomène plus récent. En revanche, le terme " intégré » suggère également que les deux types de GMN sont imbriqués l'un dans l'autre, tandis que le terme "superposé " sous-entend que la relation entre les deux types de gouvernance est peut-être moins durable.

Kettl a recours au concept des "réseaux ", tiré de la littérature sur la sociologie des réseaux, pour analyser ce qu'il appelle " la transformation de la gouvernance " dans l'administration publique et la "révolution de la gestion publique mondiale " qui a évolué depuis le début du millénaire. Il désigne les tendances accélérées vers la mondialisation et la décentralisation économiques de la fin du siècle dernier comme étant la source de l'augmentation de l'imbrication des réseaux public et privé. Comme les autres théoriciens de la GMN, il note que ces schémas de partenariats public-privé sont présents à la fois aux niveaux horizontaux et verticaux de la gouvernance et qu'ils sont un " outil essentiel pour un gouvernement hautement performant au XXI" siècle " (Kettl, 2000).

\section{La gouvernance multiniveau et le fédéralisme}

Dans un autre article (Stein et Turkewitsch, 2008a), nous avons analysé les origines et l'évolution de la GMN et les avons confrontées avec les traditions 
fédérales anglo-américaines et européennes. Depuis, nous avons appuyé de façon croissante l'utilité de ce premier concept (GMN) et l'avons appliqué à différents régimes fédéraux, matures et émergents, et à des régimes unitaires décentralisés (par exemple le Royaume-Uni) en Europe occidentale et au-delà ${ }^{4}$. Puisque notre principale préoccupation était la relation entre le fédéralisme (et les différents ordres de gouvernement) et la GMN, notre base conceptuelle était concentrée sur la GMN de type I, mais nous estimons maintenant que le concept de GMN de type II est de plus en plus utile. Les processus de la GMN de type II sont un champ que les chercheurs traditionnels du fédéralisme négligent, et nous souhaitons mettre en lumière des exemples empiriques de l'élargissement horizontal du gouvernement et sa pertinence pour la dimension administrative des relations intergouvernementales dans les régimes fédéraux.

\section{La littérature sur l'administration publique et la gouvernance multiniveau}

Un changement dans la pensée de l'administration publique est survenu dans les années 1980 et 1990 avec l'avènement de la NGP dans le monde angloaméricain (surtout en Nouvelle-Zélande, en Australie, au Royaume-Uni, aux ÉtatsUnis et au Canada) (Vining et Weimer, 2005, p. 209; Schön-Quinlivan, 2011, p. 80) et un peu plus tard dans plusieurs autres pays membres de l'OCDE (Gruening, 2001, p. 2). Même si certains chercheurs ont affirmé que l'administration publique évolue vers une phase post-NGP (Levy, 2010), nous affirmons que la comparaison entre les approches de la NGP et de la GMN est toujours pertinente. Selon Dahlström, Peters et Pierre (2011, p. 263), l'idée à la base de la NGP était d'augmenter l'efficacité du secteur public en séparant la politique des activités de prestation de services du gouvernement et de spécialiser le gouvernement. Ces changements ont mené au " décentrage " du gouvernement, c'est-à-dire qu'ils ont conduit à priver le centre de capacités de direction significatives. Par conséquent, les réformes de la NGP sont vues comme changeant le gouvernement, de la même façon que la GMN a été vue comme une puissance qui pousse les interactions gouvernementales de l'État central vers le haut à l'échelon supranational, vers le bas en direction des gouvernements régionaux et municipaux et vers l'extérieur en direction des organisations non gouvernementales et d'autres intervenants privés. Des changements similaires ont été observés dans la littérature sur l'administration publique et le fédéralisme. Ce changement d'approche dans la littérature sur l'administration publique reflète également l'émergence de l'approche de la gouvernance en science politique et dans les domaines lui étant liés 5 . Piattoni (2010, p. 20-21) fait elle aussi une analyse de la relation entre la GMN et la gouvernance en réseau, notant que les deux concepts abordent plusieurs des mêmes observations empiriques. L'élargissement horizontal de la gouvernance vers les organisations non gouvernementales et d'autres acteurs a des incidences sur l'étude de l'administration publique et représente le lien le plus fort entre la NGP et la littérature sur l'administration publique.

4 Voir Stein et Turkewitsch (2009, 2010a, 2011a et 2011b).

5 De nombreux chercheurs ont produit des historiques et des analyses sur l'émergence de l'approche de la gouvernance. Voir, par exemple, Rhodes (2000), cité dans Hooghe et Marks (2003); voir également Peters et Pierre (1998). 
Comme pour la GMN, il n'existe pas de définition de la NGP qui soit globalement attestée (Vining et Weimer, 2005, p. 209). Homburg et ses collègues (2007) ont listé les réformes les plus citées et considérées comme étant issues de la nouvelle gestion publique. Parmi elles, les plus pertinentes pour la théorisation de la GMN sont :

- les structures fortement décentralisées, comme les organismes semiautonomes, par contraste avec les grands ministères ou départements hiérarchisés à vocation multiple, ou les organismes de direction autonomes (Homburg, Pollitt et van Thiel, 2007, p. 4, citant Pollitt et autres, 2004);

- l'utilisation de structures divisionnaires dans les services publics, ce qui morcelle en définitive d'anciennes bureaucraties unitaires, ainsi que l'établissement de relations interorganisationnelles avec des organismes des secteurs privé et bénévole (PPP), ce qui finit par brouiller et étendre les frontières entre le secteur public, le secteur des entreprises soumises à l'économie de marché et le secteur bénévole et communautaire (Homburg, Pollitt et van Thiel, 2007, p. 4, citant Hacque 2001);

- un accent marqué mis sur les contrats (ou des relations de type contractuel) plutôt que sur des relations hiérarchiques et formelles (Homburg, Pollitt et van Thiel, 2007, p. 4).

De la même façon, Gruenning a recensé vingt " caractéristiques incontestées ", lesquelles sont relevées par la plupart des observateurs de la NGP : les coupes budgétaires; les pièces justificatives; l'obligation de performance; les contrôles de performance; la privatisation; [les citoyens] clients [...] ; la décentralisation; la planification et la gestion stratégiques; la séparation de la provision et de la production; la concurrence; les mesures de la performance; le changement du style de gestion; la sous-traitance; la liberté de gestion (ou flexibilité) ; l'amélioration de la reddition de comptes; la gestion du personnel (mesures incitatives); le ticket modérateur; la séparation du politique et de l'administration; l'amélioration de la gestion des finances; une plus grande utilisation des technologies de l'information (Gruenning, 2001, tableau 1).

Finalement, Homburg et ses collègues (2007, p. 5) décrivent l'approche de la NGP comme " une créature plutôt caméléonesque et paradoxale " et affirment qu'il est difficile de décrire ses caractéristiques clés. Certains chercheurs considèrent l'élaboration de politiques participative comme une caractéristique clé de la NGP (Homburg, Pollitt et van Thiel, 2007, p. 5, citant Vigoda et Golembiewski, 2001), tandis que d'autres désapprouvent cette affirmation (Homburg, Pollitt et van Thiel, 2007, p. 5, citant Gruening, 2001). Parmi les caractéristiques controversées de l'approche de NGP, Gruening (2001, p. 2) recense les contraintes légales, budgétaires et dépensières; la rationalisation des compétences; l'analyse et l'évaluation des politiques, l'amélioration de la réglementation; la rationalisation des structures administratives; la démocratisation et la participation citoyenne. En définitive, on note un chevauchement entre les caractéristiques de la NGP généralement acceptées et certaines caractéristiques controversées, ainsi que l'approche GMN dans sa définition large.

En résumé, il existe de nombreuses similitudes entre la GMN (surtout de type II) et la NGP. Les deux concepts favorisent les structures administratives décentralisées 
plutôt que hiérarchisées et accordent une plus grande place aux organisations non gouvernementales et aux PPP dans l'élaboration de politiques et leur mise en œuvre, ainsi qu'à l'élaboration participative de politiques, dont la participation citoyenne. Les deux concepts se concentrent sur la portée de plusieurs des mêmes changements empiriques survenus dans le gouvernement, les politiques publiques et l'administration publique (Piattoni, 2010). Une autre similitude est l'inexistence d'une définition largement acceptée des deux approches. La GMN et la NGP partagent d'autres points communs. Premièrement, elles sont perçues comme des constructions empiriques et des approches analytiques ${ }^{6}$. La NGP est une construction empirique définie par les changements concrets qu'elle a générés dans les gouvernements occidentaux, ainsi qu'une approche décrite dans la littérature sur l'administration publique. Gruening avance que les changements mis en œuvre par les gouvernements ont d'abord émergé et qu'ensuite les chercheurs ont recensé les caractéristiques communes de ces réformes et les ont regroupées sous l'étiquette de la NGP (Gruening, 2001, p. 2, citant Dunsire, 1995). Deuxièmement, la GMN et la NGP ont toutes deux des composantes normatives et ont soulevé des interrogations normatives ${ }^{7}$. Des chercheurs ont débattu de questions normatives liées à la GMN et à la NGP en matière de légitimité démocratique (par exemple Piattoni, 2009 et 2010), ainsi que de l'efficience bureaucratique et de l'élaboration de politiques (Vining et Weimer, 2005, p. 210). Pour Piattoni, l'une des questions normatives clés liées à la littérature sur la GMN est : « est-ce que la GMN permet une meilleure participation dans l'élaboration de politiques (légitimité des moyens) et, pour cette raison, produit de meilleures politiques (légitimité des résultats) que les autres modes de gouvernance? " (Piattoni, 2009, p. 163). Comme la GMN et la NGP s'appuient sur un plus grand apport des organisations non gouvernementales, des PPP et du secteur privé dans l'élaboration de politiques, nous présumons que la même question peut se poser quant aux processus de la NGP. Troisièmement, les deux approches ont fait l'objet de critiques; elles seraient trop descriptives ou trop empiriques ${ }^{8}$.

Une différence fondamentale entre les deux concepts est que tandis que la GMN (de type I) intègre le rôle et les interactions des différents ordres de gouvernement, la NGP ne le fait pas. Ce fait est important pour l'étude des relations intergouvernementales dans les régimes fédéraux, quasi fédéraux et unitaires décentralisés, ainsi que dans l'Union européenne, et il constitue un avantage par rapport à la NGP. Toutefois, le chevauchement entre la GMN de type I et II et l'interaction

6 Voir Stein et Turkewitsch (2010b) pour une discussion sur la GMN en tant qu'approche empirique et analytique, ainsi que sur ses composantes normatives, contrairement au fédéralisme.

7 Voir Piattoni (2010) sur les questions normatives dans la théorisation de la GMN et Vining et Weimer (2005) pour une analyse des composantes normatives dans la NGP. Hood et Peters (2004, p. 267) font également ressortir une composante normative dans la NGP, surtout lorsque celle-ci est apparue et Lewis et Marsh $(2012$, p. 6) soulignent que les concepts de la gouvernance participative et la gouvernance en réseau ont à la fois des composantes normatives et empiriques.

8 Voir Vining et Weimer $(2005$, p. 210, citant Gruening, 2001) pour des critiques de la NGP. Pour ce qui est de la GMN, Gualini (2004, cité dans Stein et Turkewitsch, 2008a, p. 10) formule cette critique du concept de la GMN. Pour un compte rendu des critiques sur le concept de la GMN, voir Stein et Turkewitsch (2008a). 
entre les deux représentent une partie importante des recherches empiriques et de la théorisation.

Le travail qu'ont réalisé Hooghe et Marks (2003) pour définir le concept de la GMN de type II est l'une des premières faisant un lien entre la littérature sur l'administration et les politiques publiques, et la théorisation de la GMN. Pour leur analyse des types I et II, ces auteurs se sont inspirés d'écrits de plusieurs domaines. Ils ont défini cinq " îlots de théorisation " (Union européenne, relations internationales, fédéralisme, gouvernements municipaux et politiques publiques), qui ont été isolés. Dans chacun de ces domaines, des chercheurs ont tenté d'expliquer la décentralisation des pouvoirs des gouvernements centraux (Hooghe et Marks, 2003, p. 234).

Peters et Pierre (1998), lorsqu'ils ont écrit sur le débat concernant la gouvernance et l'administration publique, ont également analysé les similitudes et les différences entre les approches de gouvernance et la théorie sur la gestion publique. Ils notent :

Le débat sur [...] des formes émergentes de gouvernance est apparu presque en même temps que la NGP dans plusieurs démocraties occidentales, principalement au Royaume-Uni [...] Pour certains chercheurs, le débat sur la gouvernance a été en partie lancé par la philosophie de gestion avancée par les experts de la NGP (Rhodes, 1997). De toute évidence, il y a de nombreuses similitudes entre le débat général sur les formes émergentes de gouvernance et la philosophie sousjacente à la NGP. (Peters et Pierre, 1998, p. 3)

D’une manière semblable, Ongaro et ses collègues (2010) écrivent :

La participation systématique d'une pluralité d'acteurs, parfois appelés réseaux de responsables en matière de politiques [...], qui transcende les ordres de gouvernement et les frontières du secteur public a peu à peu été considérée comme un élément de la quête pour améliorer les politiques publiques et la prestation des services que poursuivent les gouvernements. En ce sens, la notion de gouvernance est également devenue intimement liée à la NGP, même si cette dernière est beaucoup trop concentrée sur les activités internes du secteur public ou sur les instruments basés sur les mécanismes de marché. (Ongaro et autres, 2010, p. 3)

Dans la littérature américaine, des chercheurs tels que Kettl (2005) ont adopté des approches s'apparentant à la GMN. Certains travaux récents, particulièrement les deux livres dirigés par Ongaro et ses collègues (2010 et 2011), Policy, Performance and Management in Governance and Intergovernmental Relations et Governance and Intergovernmental Relations in the European Union and the United States: Theoretical Perspectives, ont tenté de lier les approches de l'administration publique et l'étude sur les relations intergouvernementales et ont mis de l'avant certains anciens liens qui avaient déjà été rapportés entre ces approches. Dans leur historique théorique des relations intergouvernementales et de la gouvernance, Ongaro et ses collègues avancent que la notion des relations intergouvernementales est largement documentée dans la littérature sur l'administration publique, surtout dans un contexte empirique et universitaire américain. Selon eux, la notion de gouvernance est aussi vieille que l'administration publique, même si la place qu'elle occupe dans la littérature est plus récente (Ongaro et autres, 2010, p. 2). 
Étant donné les zones de chevauchement entre les concepts, des chercheurs comme Osborne (2010) ont élaboré le concept de " nouvelle gouvernance publique ", qui ambitionne d'aller plus loin que la NGP et tire ses origines de la théorie sur les réseaux et les institutions. Pollitt et Bouckaert (2011, p. 22) décrivent cette nouvelle gouvernance publique comme une variante de l'approche gouvernance. Comme pour la NGP et la GMN, il n'y a pas de définition unique du concept. Toutefois, Osborne (2010, p. 9) note que « la nouvelle gouvernance publique est à la fois un résultat et une solution à la nature de plus en plus complexe et fragmentée de la mise en œuvre de politiques et de la prestation de services au XXIe siècle ». Il avance que la nouvelle gouvernance publique appelle à la fois un État pluriel dans lequel des acteurs interdépendants contribuent à la prestation de services publics et un État pluraliste dans lequel divers processus fournissent de l'information aux instances responsables de l'élaboration de politiques.

Bref, le concept de la GMN devrait être très attirant pour les chercheurs en administration publique. Il se mélange bien aux approches documentées récemment ayant des similitudes avec l'approche NGP et l'un de ses successeurs théoriques, la nouvelle gouvernance publique. Le concept de la GMN de type II est très pertinent et c'est ce que nous verrons dans les exemples suivants tirés de trois régimes.

\section{- L'ÉVOLUTION DES FORMES DE GOUVERNANCE DANS TROIS RÉGIMES FÉDÉRAUX}

Nous nous intéressons maintenant à l'apparition de tendances communes dans les régimes fédéraux australien, canadien et américain, en particulier à l'évolution des relations intergouvernementales et aux changements dans la nature de la gouvernance, la réglementation et l'élaboration de politiques. Nous pensons qu'une tendance commune s'est développée dans l'environnement politique australien, canadien et américain : un élargissement vertical en matière d'élaboration et de mise en œuvre des politiques vers les gouvernements supranationaux, étatiques ou provinciaux et municipaux, et un élargissement horizontal du processus de prise de décision et de leur mise en œuvre pour intégrer des réseaux transnationaux, des organisations non gouvernementales, la société civile, les entreprises... Nous avançons que l'examen de ces tendances peut être fait de façon optimale sous l'angle de la GMN plutôt qu'avec les autres approches traditionnelles hiérarchiques et concurrentes des relations intergouvernementales.

Plusieurs de nos exemples sont tirés de travaux de chercheurs s'intéressant aux politiques de santé publique, de travailleurs du domaine de la santé, de gestionnaires d'hôpitaux et des fonctionnaires issus du ministère de la Santé. Leur priorité étant accordée à la mise en œuvre de ces politiques, ces individus sont plus susceptibles que d'autres d'adopter un point de vue « ascendant » et semblent être également plus ouverts à l'approche GMN. Ils mettent en lumière plusieurs zones que les scientifiques politiques qui adoptent une vision plus traditionnelle et " descendante » du fédéralisme laissent souvent de côté. 


\section{L'Australie}

L'approche de la GMN est pertinente dans l'étude du fédéralisme australien, des relations intergouvernementales intérieures et des politiques publiques, particulièrement pour les politiques environnementales et de la santé. Les nouvelles formes d'interactions comprennent les réunions communautaires du cabinet, les PPP dans la gestion d'hôpitaux, les collaborations et la gouvernance en réseau dans la gestion des ressources en eau et celle des ressources naturelles dans les politiques environnementales et la cogestion et la collaboration en matière de gouvernance autochtone.

Certains spécialistes du fédéralisme australien ont été parmi les premiers à adopter le concept de GMN. Painter (2001) applique cette approche tirée d'études de l'Union européenne (et cite une définition de la GMN tirée de Marks, Hooghe et Blank, 1996) aux relations entre le gouvernement fédéral et les États australiens dans deux études de cas empiriques (enseignement et formation professionnelle, et politique de contrôle des armes à feu). Il note que les ententes institutionnelles collaboratives dans le fédéralisme australien datent du début des années 1990, bien que ces processus aient été élaborés depuis un certain nombre d'années. Il donne en exemple le New Federalism du premier ministre Bob Hawke et conclut qu'il existe peut-être une " tendance à long terme vers la diffusion et l'institutionnalisation de formes de coordination plus collaboratives dans la fédération australienne ». Son étude se concentre principalement sur la GMN de type I.

D'autres chercheurs ont continué d'analyser le succès des ententes institutionnelles collaboratives introduites par les réformes du New Federalism (par exemple Jones, 2008 et 2009). Ces dernières années, des spécialistes du fédéralisme australien ont souligné une tendance lourde vers la centralisation des pouvoirs à l'échelon fédéral et ont en même temps suggéré que le concept du " fédéralisme pragmatique " pourrait expliquer cette tendance (Hollander et Patapan, 2007, p. 281). Hollander et Patapan (2007) définissent le fédéralisme pragmatique comme étant " caractérisé par un engagement direct ou une confrontation avec des problèmes urgents, un engagement qui n'est pas modéré par des préoccupations plus grandes ». Nous pensons qu'une approche GMN peut amener les spécialistes du fédéralisme australien à orienter leurs recherches empiriques, de même que leurs analyses conceptuelles et théoriques, vers d'autres aspects des relations intergouvernementales, comme l'élargissement horizontal de la gouvernance vers des groupes du secteur privé et des organisations non gouvernementales, que l'on peut observer d'une façon optimale à travers la lunette de la GMN de type II. Or, les réformes du New Federalism ne sont pas issues de processus de GMN de type II.

Les auteurs d'articles portant sur la gestion de l'environnement, la géographie et l'administration publique ont fourni des études de cas empiriques détaillées sur les nouvelles formes de gouvernance en réseau et collaborative qui émergent dans certaines sphères du régime australien. Même si plusieurs de leurs hypothèses ont des similitudes avec l'approche GMN de type II, ces articles ne sont pas directement liés à la littérature sur le fédéralisme et la GMN.

Certains auteurs utilisent une approche qui combine les connaissances en matière de fédéralisme avec une approche de type GMN. Par exemple, Hollander 
(2009) analyse les " chevauchements " du fédéralisme australien en regard des politiques environnementales. Il cite la définition du chevauchement de Brown (1994), tirée du contexte canadien : " une situation dans laquelle il y a plus d'un gouvernement qui agit dans le même domaine politique. Plus explicitement, le chevauchement survient lorsque plusieurs ordres de gouvernement fournissent des biens et des services similaires à des clients similaires. "Certains aspects du chevauchement seront probablement mieux décrits et évalués en utilisant les concepts de la GMN. Bien que l'article de Hollander se concentre sur les réformes du fédéralisme en Australie et tente d'éliminer ces chevauchements au nom de l'efficacité et du rapport coût-efficacité, il fait remarquer que même si la fédération australienne a été soumise à des forces centripètes importantes, les États ont néanmoins continué de jouer un rôle significatif en matière d'élaboration et de mise en œuvre de politiques et que la concurrence au sein de la fédération australienne est la preuve que le régime a continué de montrer des caractéristiques du " gâteau marbré " de Grodzins ${ }^{9}$ (Hollander, 2009, p. 141). D'autres auteurs offrent des exemples similaires de collaborations continues et de chevauchements dans le fédéralisme australien.

Baracskay (2011) a adopté une approche du fédéralisme collaboratif proche de la GMN dans son étude comparative des politiques de santé en Australie, aux États-Unis et en Malaisie. Il analyse les relations collaboratives entre les différents ordres de gouvernement et les organisations non gouvernementales en regard de la mise en œuvre des politiques de santé publique et, de façon plus spécifique, la manière dont celles-ci sont gérées lors d'éclosions de maladies infectieuses. Son étude prend en compte le rôle des institutions supranationales, comme l'Organisation mondiale de la Santé, des entités régionales ainsi que des hôpitaux dans une approche qui a des similitudes avec les types I et II de la GMN. Il écrit : « [...] l'approche collaborative saisit d'une manière experte comment les différences indéfinies entre les gouvernements nationaux et supranationaux sont représentatives des aspects que d'autres dimensions de la théorie du fédéralisme ne peuvent pas toujours expliquer de façon adéquate " (Baracskay, 2011, p. 319). Baracskay estime que cette approche du fédéralisme collaboratif peut s'appliquer surtout en Australie, où il y a des responsabilités administratives, une imputabilité et une mise en œuvre partagées pour chaque ordre de gouvernement et avec les secteurs privé et bénévole.

Blanken et Dewulf (2010) comparent les PPP et les initiatives de financement privé dans les hôpitaux de l'État de Victoria et dans ceux du Royaume-Uni. L'Australie et le Royaume-Uni seraient avant-gardistes en matière de création de PPP. Il nous semble que ce type de partenariat entre des fournisseurs de soins de santé, des compagnies de gestion et de construction et des financiers soit un domaine d'études important à l'intérieur de la sphère plus large des politiques de santé et des relations intergouvernementales en Australie et au Royaume-Uni.

9 Comme nous l'avons déjà écrit (Stein et Turkewitsch, 2008b), Grodzins (2007 [1960], p. 54) affirme que le fédéralisme américain ne peut plus être décrit comme un " gâteau à trois étages », où les responsabilités sont séparées aux échelons fédéral, étatique et municipal. II propose plutôt l'expression " gâteau marbré », qui consiste en des responsabilités entrecroisées entre les différents ordres de gouvernement. 
Zurba et ses collègues (2012) se concentrent sur la gouvernance autochtone et étudient la cogestion et la gestion coopérative ou collaborative. Leur analyse conceptuelle ne s'appuie pas sur la littérature de la GMN et n'analyse pas dans le détail la structure fédérale australienne ou le système de relations intergouvernementales, mais la description qu'ils font des processus collaboratifs rappelle ceux de la NGP et de la GMN de type II. De plus, l'ajout du point de vue de la GMN permettrait d'inclure les relations intergouvernementales, y compris celles aux échelons fédéral et étatique, dans l'étude de régions indigènes protégées.

Les réunions des conseils communautaires en tant que forme de gouvernance participative sont un autre exemple de la GMN de type II en Australie (Lewis et Marsh, 2012). Cette affirmation prend le contre-pied de ceux qui soulignent une plus grande centralisation des pouvoirs sous l'égide du gouvernement national. Les conseils communautaires ont été créés par le gouvernement de l'État du Queensland en 1998 et ont ensuite été adoptés en Australie-Méridionale. Les réunions se tiennent une fois par mois et des ministres du gouvernement se rendent dans différentes régions pour y rencontrer des membres du public et discuter de problématiques locales. Les conseils communautaires ont été mis sur pied à l'échelon national en 2008. Les réunions sont ouvertes à des citoyens qui se sont préalablement inscrits. Elles commencent par un forum public d'une heure pendant lequel le premier ministre et les ministres répondent aux questions de l'assemblée. Suivent de courtes réunions organisées entre de petits groupes de participants et un seul ministre sur un sujet prédéterminé (Lewis et Marsh, 2012, p. 8). De façon générale, Lewis et Marsh restent sceptiques quant aux résultats des conseils communautaires, affirmant qu'ils ne constituent pas réellement une forme de gouvernance participative ou en réseau, mais plutôt qu'ils sont imposés de façon hiérarchique pour valider des décisions du Conseil exécutif. Peters (2008, p. 6) a également analysé ces processus de gouvernance. Il définit la métagouvernance comme la " gouvernance de la gouvernance ", une sorte de " direction " des processus informels de gouvernance sans le contrôle hiérarchique. La question du contrôle hiérarchique à l'intérieur des processus de gouvernance soulève d'importantes interrogations conceptuelles concernant la GMN, y compris la relation entre les types I et II, que nous tenterons de régler dans notre analyse finale et nos conclusions.

Benson et Jordan (2010) se concentrent sur la division des problématiques dans la politique sur l'eau dans une analyse comparative entre l'Union européenne et l'Australie. Leur recherche offre des descriptions empiriques de la politique sur la qualité de l'eau d'Australie qui suggèrent que l'approche GMN est de plus en plus appropriée dans ce cas. Ils écrivent qu'en Australie, la division de la gouvernance de l'eau a évolué d'une situation de départ où les tâches étaient (pour la plupart) dévolues à des ordres inférieurs de gouvernement à une situation de partage des tâches entre les gouvernements. Benson et Jordan (2010) utilisent une approche comparative du fédéralisme qui intègre également des éléments de l'approche GMN tirée à la base du contexte européen et constitue donc un exemple de l'approche plutôt synthétique que nous défendons. De plus amples recherches dans cette lignée seraient bénéfiques. Par ailleurs, Prager (2010) se concentre sur 
des réseaux collaboratifs ${ }^{10}$, dont les entités de gestion des ressources naturelles australiennes, qui sont similaires aux processus de la GMN de type II. Son article donne des exemples empiriques de processus de gouvernance qui pourraient être d'intérêt pour les chercheurs en GMN. Par contre, il ne cite ni ne touche théoriquement de façon explicite la littérature sur la GMN ou le fédéralisme.

Dans une autre étude empirique, Head (2011) analyse la participation citoyenne et la consultation populaire en Australie. Dans l'une de ses quatre études de cas se concentrant sur les politiques environnementales, il fait remarquer que les processus faisant appel à de multiples acteurs en environnement et en gestion des ressources naturelles sont devenus courants en Australie ces vingt dernières années (Head, 2011, p. 108-109). Il affirme que certains domaines politiques, comme la politique étrangère, sont moins favorables à la participation citoyenne puisqu'ils sont plutôt techniques ou caractérisés par des processus de prise de décision fermés. Head avance que la consultation populaire est de plus en plus ancrée dans plusieurs domaines politiques clés comme les services publics en milieu urbain et la gestion de l'environnement. En revanche, il considère que dans plusieurs cas, la participation citoyenne est purement symbolique. Ces différences entre les domaines politiques sont importantes pour les théoriciens de la GMN.

\section{Le Canada}

La présente sous-section propose des exemples empiriques de processus de GMN de type II au Canada, majoritairement tirés des politiques environnementales et de santé publique ${ }^{11}$. Nous donnons également des exemples de coopération transfrontalière entre le Canada et les États-Unis, qui constituent des exemples de processus de GMN de type II en action.

En matière de politique de santé publique au Canada, les réseaux de santé des Premières Nations sont un exemple de gouvernance multiniveau en ce qui a trait à l'élargissement à la fois vertical et horizontal de la gouvernance. Substantiellement, ils peuvent également constituer un exemple de chevauchement de GMN de type I et II, comme le décrivent Leo et Enns (2009, p. 94). Les spécialistes du fédéralisme canadien et les étudiants en politiques autochtones au Canada adoptent de plus en plus le point de vue de la GMN (par exemple Papillon, 2008); toutefois, peu de travaux sont directement liés aux politiques de santé publique. Une exception à cet état de fait est la recherche réalisée par Smith et Lavoie qui décrit les réseaux de santé des Premières Nations, dont chacun réunit plusieurs nations autochtones sous l'autorité d'un conseil de bande, d'un comité de planification ou d'une autorité en matière de santé publique (Smith et Lavoie, 2008, p. 101-102). Le programme Transfert canadien en matière de santé mis en œuvre par le gouvernement fédéral en 1987 offre aux communautés des Premières Nations de la flexibilité en matière d'autogouvernance (Smith et Lavoie, 2008, p. 102). Les ententes de transfert avec

\footnotetext{
${ }^{10}$ L'expression « réseau collaboratif » utilisée par Prager est synonyme de " partenariat ». Les réseaux collaboratifs sont composés de parties prenantes qui représentent leur communauté ou diverses organisations (Prager, 2010, p. 710).

${ }^{11}$ Voir Stein et Turkewitsch (2011a) pour une discussion sur les relations intergouvernementales et une analyse des processus de GMN de type I au Canada et aux États-Unis.
} 
les Premières Nations et les communautés inuites portent sur les soins de santé à différents échelons de la gouvernance : à l'échelle de la communauté, où l'accent est mis sur la prestation directe de services; à l'échelle du secteur, là où s'effectuent la coordination et la supervision; à l'échelle régionale, là où ont cours les consultations et où se formulent les recommandations; à la direction centrale, sous l'autorité de la Direction générale de la santé des Premières Nations et des Inuits de Santé Canada, à l'échelle fédérale (Smith et Lavoie, 2008, p. 103). Smith et Lavoie (2008) décrivent la mise en œuvre par les Premières Nations de réseaux collaboratifs, qui comprennent de nombreuses communautés rassemblées à travers des autorités en matière de santé ou des conseils de bande.

Elson (2005) fait également ressortir l'existence d'un aspect horizontal de la GMN, qu'il décrit comme étant un partage informel de pouvoirs, au sein du ministère de la Santé de l'Ontario. Des acteurs des secteurs privé, public et non gouvernemental, en particulier l'Ontario Medical Association, l'Ontario Hospital Association, ainsi que les grands hôpitaux universitaires de la province ont en partie influencé les décisions en matière de politique de santé publique (Elson, 2005). Nous pensons que la GMN en tant que structure conceptuelle peut être utile pour analyser le rôle des entités régionales et locales de gestion des soins de santé au sein des provinces canadiennes, de même que l'influence des acteurs des secteurs privé, non gouvernemental ou public.

L'approche GMN invite également les chercheurs à se pencher sur l'échelon local à l'intérieur du système fédéral. Au Canada, on note de plus en plus de partenariats trilatéraux entre le fédéral, le provincial et le municipal. Un exemple de ces partenariats, qui fait également ressortir le rôle du gouvernement local dans les soins de santé, est l'Accord de Vancouver signé en 2000 (Bradford, 2005, p. 41). Cet accord a réuni un nombre impressionnant d'acteurs de tous les niveaux, dont douze ministères fédéraux, dix-neuf ministères et agences provinciaux, le Département de police de Vancouver et la Vancouver Coastal Health Authority. L'Accord de Vancouver met l'accent sur l'amélioration de la santé publique dans le centre urbain comme base de la régénération économique et communautaire. L'approche gouvernance multiniveau offre aux chercheurs une motivation pour se pencher davantage sur la coordination politique dans les partenariats trilatéraux.

L'approche GMN peut également se révéler utile pour comprendre d'autres innovations en matière de politiques de santé publique dans les provinces canadiennes. Un exemple d'une telle innovation est la régionalisation de la gestion des soins de santé. Contandriopoulos et ses collaborateurs (2004) parlent de cette régionalisation comme de la " création d'une nouvelle structure organisationnelle d'autorité afin d'augmenter les capacités stratégiques de l'échelon intermédiaire " (Denis, 1999; Denis et Valette, 2000, cités dans Contandriopoulos et autres, 2004, p. 629). Ils suggèrent que même si la régionalisation est souvent considérée comme un équivalent de la décentralisation, elle est mieux décrite comme étant une forme complexe de décentralisation couplée à des éléments de centralisation. Faisant partie des réformes des soins de santé des années 1990, les réformes de régionalisation ont été implantées dans neuf provinces afin d'atteindre des objectifs financiers. Contandriopoulos et ses collègues se sont penchés sur le rôle des 
régies régionales de la santé et des services sociaux au Québec du point de vue de la gestion publique. Ils considèrent la régionalisation comme une forme de renouvellement de la gouvernance des systèmes provinciaux de soins de santé. D'une façon similaire, en Ontario, les directions régionales de santé et les réseaux locaux d'intégration des services de santé existent en tant qu'avenues possibles pour la décentralisation des pouvoirs provinciaux du ministère de la Santé et des Soins de longue durée, surtout en ce qui concerne la gestion et la gouvernance de la politique de soins de santé (Elson, 2005).

De nombreux exemples de gestion environnementale participative au Canada et aux États-Unis peuvent également être analysés grâce à la GMN. Newig et Fritsch (2009) ont étudié quarante-sept cas de gestion environnementale participative en Europe occidentale et en Amérique du Nord dans la littérature sur la gestion publique collaborative. Leur étude vise à évaluer l'efficacité des systèmes de GMN, et elle nous est utile car elle réunit une liste de cas aux États-Unis et au Canada qui peuvent être analysés au moyen de la GMN de type II. Comme le font remarquer Newig et Fritsch (2009), il existe de nombreux exemples de l'élargissement horizontal de la gouvernance comme l'augmentation de la participation citoyenne dans l'assainissement des Grands Lacs. Dans le cas de la rivière Ashtabula en Ohio et du port de Collingwood, sur le lac Ontario, des groupes locaux ont collaboré à améliorer les conditions environnementales afin de soutenir la croissance économique des secteurs de la pêche et du tourisme. Dans le cas du port de Collingwood, des organismes gouvernementaux des différents ordres de gouvernement, dont Environnement Canada, le ministère ontarien des Ressources naturelles et celui de l'Environnement et de l'Énergie, ont participé. Des organisations non gouvernementales et des groupes communautaires ont également apporté leur contribution (Krantzberg et Houghton, 1996). Pour la rivière Ashtabula, la baie de Quinte et le port de Collingwood, on a mis à contribution des acteurs locaux, régionaux et supranationaux ainsi que des " processus participatifs dans un contexte de gouvernance multiniveau » (Newig et Fritsch, 2009, p. 207).

En plus de ces mouvements locaux, les ententes transnationales entre le Canada et les États-Unis, de même que le système émergent de " gouvernance transfrontalière des Grands Lacs ", constituent d'autres exemples de GMN en matière de restauration environnementale des Grands Lacs (Karkkainen, 2004). En 1972, le Canada et les États-Unis ont négocié l'Accord relatif à la qualité de l'eau dans les Grands Lacs qui fixe des objectifs de qualité de l'eau et des mesures coordonnées pour contrer la pollution que les deux pays mettent en œuvre à travers leurs lois nationales de lutte contre la pollution. En 1978 et en 1987, des modifications apportées à l'Accord ont établi comme cible l'atteinte d'un " écosystème intégré de gestion " des Grands Lacs (Karkkainen, 2004). Outre les gouvernements fédéraux du Canada et des États-Unis, les acteurs de ce système se trouvent au sein des gouvernements infranationaux de chacun des pays, d'organisations intergouvernementales et non gouvernementales et de la société civile, formant un " réseau transfrontalier de coopération régionale " (Karkkainen, 2004). Sont parmi les participants, les huit États américains et les deux provinces canadiennes qui bordent les cinq lacs, des représentants des principaux ports et des municipalités importantes de la région, des gouvernements des peuples autochtones des États-Unis et des Premières Nations 
du Canada, des organisations intergouvernementales régionales, des organisations non gouvernementales environnementales, des organisations sportives et de pêcheurs, des associations d'entreprises et des chambres de commerce, ainsi que des experts scientifiques. Selon Karkkainen, ces « ententes de gouvernance polycentrique " reposent sur :

[...] une collaboration transfrontalière soutenue et constante entre plusieurs acteurs étatiques et non étatiques engagés dans la cogestion d'une ressource partagée [...] Les accords institutionnels qui définissent les efforts communs de gestion vont bien au-delà des organes de chacun des deux pays souverains afin d'inclure des acteurs infranationaux et étatiques, agissant à la fois dans les canaux formels et informels - et parfois extraconstitutionnels - pour rendre la gouvernance effective. (Karkkainen, 2004, p. 90)

Il existe de nombreuses similitudes entre l'analyse de la gouvernance polycentrique de Karkkainen et les travaux de Hooghe et Marks sur la GMN de type II.

En plus des rôles que les municipalités ont joués dans les processus collaboratifs décrits auparavant, on voit apparaître de nombreuses initiatives à l'échelle des villes dans le domaine des politiques environnementales et des changements climatiques au Canada et aux États-Unis. Comme l'écrivent Gore et Robinson (2009, p. 137), depuis la fin des années 1980, les gouvernements municipaux en Amérique du Nord sont apparus comme des chefs de file en matière de réponse aux changements climatiques. Ils sont devenus d'importants acteurs dans un système multiniveau de gouvernance des changements climatiques. Toutefois, les approches traditionnelles du fédéralisme et les relations intergouvernementales ont systématiquement fait fi du rôle des gouvernements municipaux, qui sont vus comme des " créatures " d'une province ou d'un État.

Au Canada comme aux États-Unis, les villes ont bâti un réseau national municipal, réseau qui s'est attaqué à la problématique des changements climatiques. Au Canada, la plupart des villes sont membres de la Fédération canadienne des municipalités, et aux États-Unis, de nombreuses villes ont adhéré à la Conference of Mayors (Gore et Robinson, 2009, p. 141). Les deux réseaux sont partenaires de réseaux transnationaux environnementaux et de lutte contre les changements climatiques. Le Conseil international pour les initiatives écologiques communales, une association internationale de gouvernements municipaux, est parmi les principaux réseaux du genre (Gore et Robinson, 2009, p. 140). La Fédération canadienne des municipalités a travaillé de concert avec le Conseil dans le cadre du programme Partenaires dans la protection du climat (Bradford, 2005, p. 35). Au Canada, les Fonds municipaux verts du gouvernement fédéral permettent à la Fédération d'investir dans des technologies touchant des questions environnementales comme la gestion des déchets et le traitement de l'eau.

\section{Les États-Unis}

Aux États-Unis, une vision plus large du fédéralisme et des relations intergouvernementales, proche de celle de la GMN, est de plus en plus adoptée et soutenue par les chercheurs des domaines de l'administration et la gestion publiques (Kettl, 2000; Hoflund et Farquhar, 2008), du droit (Trubek, 2006; Blum, 2008) et 
de la sociologie (Heimer et autres, 2008), de même que par les spécialistes des politiques au sein d'agences gouvernementales (Hoflund et Farquhar, 2008). Nous pensons que ces chercheurs sont plus enclins que les spécialistes du fédéralisme traditionnel à adopter une approche semblable à celle de la GMN. Nous nous appuyons sur des exemples tirés d'écrits sur les politiques environnementales et la santé publique qui montrent qu'une approche GMN est de plus en plus utile pour les chercheurs en administration publique et en fédéralisme lorsque vient le temps d'analyser les structures changeantes des relations intergouvernementales et les processus d'élaboration de politiques. Dans le domaine des politiques environnementales, nous citons également des exemples comparatifs tirés du contexte canadien.

La littérature récente portant sur les politiques comparatives en matière d'environnement (Durant, 2004; Durant, Fiorino et O'Leary, 2004; John, 2004; Scheberle, 2004a et 2004b; Schlager, 2004), de nouvelle gestion publique et d'organismes publics (Newig et Fritsch, 2009) et de droit de l'environnement et des ressources naturelles (Karkkainen, 2004) est plus ouverte qu'avant à prendre en considération de nouvelles initiatives en matière de GMN. De plus, des chercheurs d'autres domaines, comme la géographie (Bulkeley, 2006; Bulkeley et Moser, 2007) et l'urbanisme (Gore et Robinson, 2009) ont publié des études à " petit $\mathrm{n}$ " sur la gestion locale environnementale et ont analysé de nouveaux processus dans la gouvernance urbaine à partir de points de vue qui ressemblent à ceux de l'approche GMN ${ }^{12}$.

Trubek (2006) étudie la "nouvelle gouvernance " et le " droit mou " dans les soins de santé aux États-Unis. Sa définition de la nouvelle gouvernance est très semblable à celle de la GMN que nous avons mise en relief plus tôt ${ }^{13}$. Elle fait observer que la nouvelle gouvernance comprend la décentralisation du gouvernement, les PPP, de nouveaux types de réglementations et d'incitatifs, la création de réseaux, la coordination de collectes de données et de la diffusion d'analyses comparatives, la vérification et l'engagement personnel actif. De plus, elle note que la décentralisation déplace les pouvoirs vers les ordres inférieurs de gouvernement et réduit l'accent mis sur les programmes inflexibles administrés à l'échelle nationale. Il existe une interaction multiniveau au sein de laquelle le gouvernement national fixe les standards ou fournit le financement accompagné d'un lien entre les ordres de gouvernement fédéral, étatiques et municipaux (Trubek, 2006). S'agissant de l'élargissement horizontal de la gouvernance, Trubek considère les PPP et le recours à la négociation par les réseaux comme des attributs de la "nouvelle gouvernance ». Elle fournit plusieurs exemples de nouvelle gouvernance dans les

12 Voir, par exemple, des travaux récents dans des revues comme Local Environment, Climate Policy et Climatic Change, qui fournissent une mine de cas qui n'ont pas été traités par les études traditionnelles sur le fédéralisme.

${ }^{13}$ Trubek apporte également une perspective GMN à l'étude de la politique de santé publique des États-Unis grâce à ses articles sur la «nouvelle gouvernance » dans I'Union européenne, ou la méthode de coordination ouverte. Ceci est un exemple intéressant de " pollinisation croisée » entre les études sur l'Union européenne et celles sur les politiques américaines (Trubek et Trubek, 2005). Son approche partage également de nombreuses similitudes avec la littérature sur les « nouveaux modes de gouvernance ", en particulier celle qui se concentre sur l'Union européenne (voir, par exemple, I'ouvrage dirigé par Héritier et Rhodes, 2011. 
politiques de soins de santé des États-Unis au milieu des années 2000. Ceux-ci sont également pertinents pour l'analyse faite à l'aide de la lunette conceptuelle de la gouvernance multiniveau. Les nouveaux espaces collaboratifs dans lesquels les parties prenantes sont engagées pour régler des problématiques concernant les soins de santé constituent l'un de ces exemples. Ces espaces peuvent être des réseaux collaboratifs de contrôle de la qualité, des groupes de technologie locaux et des planifications de groupe pour mettre en commun les régimes de couverture publics et privés (Trubek, 2006). Les réseaux collaboratifs sont institués aux échelons local, national et étatique.

Une autre initiative pouvant être considérée sous l'angle de la GMN de type II est le National Quality Forum qui, comme l'écrivent Hoflund et Farquar, rassemble des parties prenantes du domaine des soins de santé des secteurs public et privé afin qu'ils s'attaquent aux questions de qualité et de rendement. Le Forum compte plus de trois cent soixante-dix membres de différents « secteurs de l'industrie des soins de santé, dont des acheteurs, des clients, des fournisseurs et des organismes d'amélioration de la qualité " (Hoflund et Farquar, 2008, p. 121).

Il existe également des exemples de l'élargissement externe de la gouvernance en matière de politique de soins de santé aux échelons local et étatique, comme les réseaux d'agences de soins de santé et de services sociaux communautaires, qui ont des attaches avec les agences gouvernementales fédérales ou des États (Huang et Provan, 2006). Par exemple, en 1992, l'Arizona a sous-traité ses services en matière de santé mentale à cinq agences régionales de santé comportementale (Regional Behavioral Health Authorities). Ces agences ont été régies à un moment donné par des organismes sans but lucratif ou des entreprises privées de soins (Huang et Provan, 2006). Huang et Provan (2006) et Provan, Huang et Milward (2009) se sont penchés sur un réseau d'agences de soins de santé et de services sociaux communautaires en Arizona, bien que leur préoccupation première soit l'analyse de réseaux, analyse qui néglige les composantes intergouvernementales. D'un autre côté, les approches traditionnelles du fédéralisme ne tiennent pas compte de ce genre de réseaux. De notre point de vue, il faut une approche plus intégrée, comme la gouvernance multiniveau, qui prend en considération à la fois des dynamiques intergouvernementales verticales et les caractéristiques horizontales de ces réseaux.

Il est peut-être trop tôt pour apprécier l'importance des relations intergouvernementales liées aux réformes des soins de santé de l'administration Obama, lesquelles ont été promulguées en tant que loi (l'Affordable Care Act) le 23 mars 2010. Toutefois, la tendance à l'augmentation de la participation d'acteurs non gouvernementaux, des PPP, des coalitions pour la qualité et d'autres groupes semble vouloir persister sous cette loi. Selon nous, l'approche GMN est utile pour analyser ces changements puisqu'elle explore des perspectives plus larges que les autres approches peuvent omettre.

En ce qui concerne la littérature nord-américaine sur les sciences politiques, les spécialistes du fédéralisme emploient de plus en plus l'expression "gouvernance multiniveau ", quoiqu'ils utilisent cette dernière et le terme "fédéralisme " 
de manière interchangeable ${ }^{14}$. D'autres ont appliqué le concept de la GMN et ont apporté des améliorations théoriques à l'approche. Par exemple, pour ce qui est des politiques en matière de changements climatiques en Amérique du Nord, Selin et VanDeveer (2009a) se sont penchés sur l'engagement d'ordres de gouvernement multiples dans l'élaboration de politiques, de même que sur de nouvelles formes de partenariats entre acteurs du privé, du public et de la société civile. Ils définissent la GMN comme des acteurs opérant à la fois sur les dimensions verticales et horizontales d'organismes sociaux autour d'une problématique donnée (Selin and Vandeveer, 2009c, p. 316). Selon eux, la gouvernance en matière de changements climatiques en Amérique du Nord implique des interactions importantes entre les responsables politiques municipaux, provinciaux, étatiques et fédéraux, des leaders du secteur privé et des représentants de la société civile. Toutefois, ils avancent également que " contrairement à la gouvernance multiniveau dans l'Union européenne, les politiques nord-américaines en matière de changements climatiques ne reposent pas sur un leadership fort du gouvernement national ou sur des efforts soutenus pour coordonner les politiques entre les États " (Selin et VanDeveer, 2009b, p. 7). Ils soutiennent que la gouvernance en matière de changements climatiques au Canada et aux États-Unis a été principalement marquée par des " dynamiques ascendantes ». Nous croyons qu'il s'agit d'une différence analytique clé, et nous aborderons la signification des initiatives ascendantes et des pressions descendantes dans la théorisation de la GMN et nos conclusions.

Le programme de préservation de la baie Chesapeake, cité par de nombreux auteurs (Costanza et Greer, 1995; Karkkainen, 2004; Posner, 2009), est un exemple d'une nouvelle forme de gouvernance collaborative et il est compris dans une perspective de GMN. Ce programme est un partenariat de gestion collaborative des lignes de partage des eaux (Posner, 2009) du District de Columbia, des États limitrophes de la baie, de l'Environmental Protection Agency et d'autres agences fédérales, ainsi que des groupes de pression sans but lucratif et des gouvernements municipaux de la région. Le mouvement s'est d'abord constitué en tant que réseau comprenant des sociétés gouvernementales, sans but lucratif et privées qui ont collaboré afin de nettoyer la baie (Posner, 2009). Il s'est par la suite institutionnalisé sous la forme du programme de préservation de la baie Chesapeake. Posner (2009) considère ce programme comme un exemple de " gouvernance environnementale collaborative " et Karkkainen (2004) le perçoit comme un exemple de " gouvernance environnementale postsouveraineté " qui fait partie de " l'écosystème des ententes de gouvernance les plus soutenues, matures, complexes institutionnellement et réussies " (Costanza et Greer, 1995, cités dans Karkkainen, 2004, p. 80). Dans son article sur la gouvernance postsouveraineté, Karkkainen note que dans le domaine des politiques environnementales, les États-Unis passent

\footnotetext{
${ }^{14}$ Par exemple, dans son article sur les politiques en matière de changements climatiques dans un système multiniveau, Rabe (2007) cite les États-Unis et le Canada comme étant des systèmes de gouvernance multiniveau, mais ne parle pas plus en détail de cette théorie. En revanche, dans un autre article, Rabe est plus enclin à parler de l'approche et de la terminologie de la « gouvernance en réseau ", comme il l'a fait dans son analyse d'une " gouvernance en réseau pour le climat en Californie » (Rabe, 2009a, p. 46).
} 
rapidement d'un modèle régulateur traditionnel axé sur la souveraineté basé sur une réglementation de type hiérarchique, à la pièce et impérative, à un modèle axé sur des ententes de gouvernance polyarchique expérimentale, collaborative, largement intégrante et taillée pour une région ou une localité (Dorf et Sabel, 1998 et Sabel et autres, 2000, cités dans Karkkainen, 2004, p. 78).

Il existe d'autres exemples de l'élargissement externe de la prise de décision dans le domaine des politiques environnementales qui concernent des acteurs non gouvernementaux, des groupes de la société civile et des entreprises privées, ainsi que de l'élargissement vertical qui concernent différents ordres de gouvernement. Durant et ses collègues prennent l'exemple du Colorado Plateau Ecosystem Partnership Project. Ce projet a été mis en place par l'Environmental Protection Agency et le National Park Service, qui ont également fait appel à des partenaires gouvernementaux et non gouvernementaux, dont des agences fédérales, des gouvernements d'États, des nations amérindiennes, des comités gouvernementaux et des entreprises privées du Colorado et des États voisins (Utah, Arizona et NouveauMexique) (Durant, Fiorino et O'Leary, 2004, p. 15).

Une autre initiative dans le domaine des politiques régionales sur les changements climatiques élaborée par des groupes étatiques et provinciaux est la Regional Greenhouse Gas Initiative. Fondée en 2009, cette initiative a établi un dispositif régional de plafonnement et d'échanges de quotas d'émissions de dioxyde de carbone provenant des centrales électriques d'un regroupement de dix États du nord-est du pays (Selin et VanDeveer, 2009c, p. 120-121). Plusieurs provinces canadiennes, dont les délégués assistent aux réunions à titre d'observateurs, y participent également. Les règles que les participants doivent suivre sont inscrites dans un " règlement modèle " rédigé selon un processus collaboratif et tenant compte des commentaires des producteurs d'énergie de la région, de groupes de la société civile et d'entreprises privées (Selin et VanDeveer, 2009c, p. 121).

Enfin, un autre exemple qui se prête particulièrement bien à l'analyse selon une perspective GMN est la participation de villes canadiennes et américaines à la campagne Des villes pour la protection du climat, « un réseau à la fois mondial et local, étatique et non étatique " (Betsill et Bulkeley, 2006, p. 141). Betsill et Bulkeley avancent qu'une perspective multiniveau est particulièrement utile pour comprendre cette campagne, car les gouvernements infranationaux et leurs réseaux agissent comme des acteurs transnationaux indépendants. L'approche GMN peut être un point de départ utile pour commencer l'analyse.

\section{- ANALYSES COMPARATIVES ET CONCLUSIONS}

Les exemples présentés montrent qu'une démarche de gouvernance multiniveau fait ressortir de nouvelles formes d'élaboration et de mise en œuvre de politiques qui incorporent l'élargissement horizontal et vertical de la gouvernance, élargissement que les approches traditionnelles du fédéralisme ne prennent souvent pas en compte. L'approche GMN se combine également très bien avec d'autres approches récentes en administration publique, dont la nouvelle gestion publique et la nouvelle gouvernance publique, en raison de leurs similitudes et 
des liens théoriques qu'elles partagent avec l'approche de gouvernance en général. Plusieurs de ces nouvelles formes de gouvernance ne sont pas des processus descendants typiques dans lesquels le gouvernement fédéral (ou provincial/étatique) joue un rôle prédominant en matière de régulation. Elles sont plutôt basées sur des ententes volontaires entre différents acteurs, dont les gouvernements municipaux, les organisations non gouvernementales, des entreprises privées et la société civile. Certains processus collaboratifs sont des initiatives ascendantes. Les partenariats régionaux et transfrontaliers qui font appel à divers ordres de gouvernement, ainsi qu'à des groupes de la société civile et d'autres acteurs, ne sont également pas pris en compte par les approches traditionnelles du fédéralisme, mais se prêtent bien à l'analyse selon une perspective GMN.

Une question importante à considérer est de savoir si des méthodes d'élaboration de politiques ou des pressions " descendantes " aident à mettre en place des initiatives " ascendantes " ou les découragent, et si des initiatives ascendantes favorisent ou font obstacle aux initiatives descendantes ${ }^{15}$. Dans les approches traditionnelles du fédéralisme, une structure hiérarchique va généralement de soi, ce qui limite le rôle d'initiatives ascendantes. Pourtant, la question du contrôle opéré par le gouvernement central par rapport aux pressions exercées par les gouvernements infranationaux pour la décentralisation est un débat classique dans la littérature et un sujet de tension dans certains régimes fédéraux et unitaires. Dans l'approche GMN, l'interaction entre les différents ordres suppose que les initiatives ascendantes émanant du municipal ou du provincial/étatique peuvent avoir une influence. De plus, les gouvernements centraux peuvent faire l'objet de pressions descendantes d'un gouvernement supranational, comme l'Union européenne. Ces pressions risquent de limiter encore plus le rôle que jouent les gouvernements centraux dans l'élaboration de politiques.

Mais les relations entre le gouvernement central, ou le gouvernement supranational, et les ordres infranationaux sont-elles collaboratives ou litigieuses? Nous serions portés à affirmer que la réponse à cette question dépend grandement des actions et des motivations de l'échelon supérieur. La théorie de la GMN suppose une relation collaborative entre les différents ordres de gouvernement, et entre les acteurs d'une GMN de type II issus de l'élargissement horizontal de la gouvernance. Le fédéralisme et les autres approches théoriques peuvent prévoir des relations quelque peu litigieuses. En effet, les pressions descendantes exercées afin de bloquer les pressions ascendantes peuvent faire face à une forte opposition des ordres inférieurs de gouvernement et d'autres acteurs.

La question des pressions descendantes et ascendantes touche également la perspective théorique ou situationnelle. Par exemple, comme nous l'avons écrit précédemment, les praticiens du domaine des politiques de santé publique tendent à présenter une approche ascendante, tandis que les spécialistes du fédéralisme ont plutôt tendance à se concentrer sur les échelons du haut et à choisir une perspective descendante. Entre ces deux extrêmes, l'approche GMN (qui intègre les types I et II) constitue un juste milieu. De plus, elle incite les chercheurs

${ }^{15}$ Nous remercions le lecteur critique anonyme de Télescope d'avoir soulevé cette question. 
à tenir compte des pressions ascendantes plutôt que descendantes, ce que les approches de la nouvelle gestion publique ne font pas. Les interactions descendantes et ascendantes sont également importantes pour la GMN de type I et, en ce sens, il s'agit d'un domaine pour lequel il est préférable d'opter pour cette dernière plutôt que pour les approches traditionnelles du fédéralisme.

Nous sommes d'accord avec Howlett (2000, p. 305) qui soutient qu'en matière de politiques environnementales le Canada et les États-Unis «n'avancent pas l'un vers l'autre, mais tendent plutôt vers une approche tierce, commune, qui se base sur la mise en œuvre d'initiatives autorégulées et volontaires, influencées par les idées et principes de la nouvelle gestion publique ". De la même façon, nous adhérons aux opinions de Huque et Watton (2010, p. 83, citant Valiante, 2002, p. 16) qui affirment que le Canada et les États-Unis vivent un « éloignement des contrôles et régulations gouvernementaux [...] et tendent vers un cadre d'initiatives volontaires du secteur privé et d'autorégulation » dans le domaine des politiques environnementales. Ils soutiennent que les deux pays adoptent un système dominé par les principes du volontariat, de la privatisation et de la marchandisation. Même si l'Australie n'a pas été prise en compte dans les comparaisons faites par ces auteurs, les exemples que nous avons donnés suggèrent que des processus similaires sont engagés dans le domaine des politiques environnementales en Australie. L'approche GMN sera utile pour conduire des analyses comparatives des processus intergouvernementaux dans ces trois fédérations.

On note un changement de dynamique dans le secteur de la santé en Australie, au Canada et aux États-Unis. Les exemples cités précédemment (les PPP pour la gestion d'hôpitaux et les relations collaboratives entre les différents ordres de gouvernement et les organisations non gouvernementales en Australie; le réseau des conseils de santé des Premières Nations, l'Accord de Vancouver et les réseaux locaux d'intégration des services de santé au Canada; le National Quality Forum, d'autres réseaux collaboratifs locaux de contrôle de la qualité et de nouvelles formes de gouvernance par des agences gouvernementales aux États-Unis) sont des indicateurs de tendances plus générales et du changement de la nature de la gouvernance et de la régulation, non seulement dans les domaines des politiques de santé et de l'administration, mais également dans d'autres domaines politiques. Encore une fois, l'approche GMN peut être pratique pour étudier les politiques de santé publique, car elle met l'accent sur l'élargissement vertical et horizontal de la prise de décision intergouvernementale.

En somme, le concept de la gouvernance multiniveau, de même que des idées tirées de la nouvelle gestion publique et de la nouvelle gouvernance publique, est pertinent pour la recherche sur le fédéralisme et les relations intergouvernementales ainsi que pour celle sur l'administration publique et les sciences politiques. En rassemblant des études de ces différents domaines, le concept de la GMN peut faire ressortir les formes de gouvernance émergentes dans les régimes fédéraux et unitaires décentralisés. Dans sa définition même, la GMN couvre à la fois l'élargissement horizontal de la gouvernance (préoccupation première d'une grande partie des chercheurs en administration publique et en politiques environnementales et de santé) et les ordres verticaux de gouvernement dans les régimes 
fédéraux ou unitaires décentralisés (préoccupation centrale des étudiants en relations intergouvernementales).

Malgré de nombreuses similitudes entre les contextes australien, canadien et américain, il existe des différences entre les processus de GMN selon les domaines politiques et la conjoncture nationale. Les différences de structures institutionnelles et les différents points d'accès pour les organisations non gouvernementales et autres acteurs sont des facteurs pouvant modeler l'émergence de processus de GMN de type II. De plus amples recherches comparatives nous permettront d'affiner la définition de la GMN et d'étendre son utilité en tant que cadre d'analyse. Cet article s'inscrit dans un projet de recherche plus large sur la GMN dans un contexte comparatif, et nous invitons les lecteurs à consulter nos écrits antérieurs sur ce sujet dans d'autres contextes, ainsi que notre ouvrage à paraître ${ }^{16}$ qui pousse plus loin cette recherche.

La question du contrôle hiérarchique à l'intérieur de la gouvernance en réseau est soulevée dans plusieurs exemples. L'argument de Lewis et Marsh (2012) selon lequel les conseils communautaires en tant que processus participatif sont imposés hiérarchiquement pour valider des décisions de l'exécutif repose non seulement sur la nature même des processus de la GMN de type II, mais également sur les questions normatives de légitimité démocratique, une problématique dont nous ne pouvons pas tenir compte dans cet article par manque d'espace. Même si par définition un réseau ne contient aucun élément hiérarchique, nous pensons qu'il peut être difficile d'en trouver un exemple dans les contextes utilisés dans notre étude. La GMN en soi est également définie comme s'éloignant des processus hiérarchiques de la prise de décision; par contre, même dans ce cas, un élément de contrôle hiérarchique peut demeurer.

L'aspect du chevauchement entre les processus de type I et II de la GMN est pertinent pour l'approfondissement du concept de la GMN. Nous croyons que de nouvelles recherches sont nécessaires. Comme abordé précédemment, un débat a cours sur la question de savoir de quelle façon la relation entre les deux concepts devrait être décrite - chevauchement, multicouche ou imbrication. Les types I et II de la GMN devraient-ils demeurer distincts analytiquement ou devraient-ils être combinés comme le suggèrent Leo et Enns (2009)? Nous sommes d'avis que la distinction faite entre les deux types de GMN est utile sur le plan analytique et sert de référence pour la question de l'élargissement vertical par rapport à l'élargissement horizontal. Le recoupement entre les deux types et la forme de coexistence entre eux sont peut-être la zone de recherche la plus significative pour les spécialistes du fédéralisme et des relations intergouvernementales et pour les spécialistes de l'administration publique et de domaines politiques précis. C'est également la zone où ces disciplines se partagent le plus de terrain. C'est là que le concept de la GMN peut servir comme concept unificateur et combler les fossés disciplinaires.

${ }^{16}$ Multi-Level Governance and Federalism: Analyzing Intergovernmnetal Relation, à paraître chez Edward Elgar. 


\section{BIBLIOGRAPHIE}

Bache, I. (2008). Europeanization and Multi-level Governance: Cohesion Policy in the European Union and Britain, Lanham, Rowman \& Littlefield.

Baracskay, D. (2011). " How Federal Health-Care Policies Interface with Urban and Rural Areas: A Comparison of Three Systems ", Global Public Health: An International Journal for Research, Policy and Practice, vol. 7, n 4, p. 317-336.

Benson, D. et A. Jordan (2010). « The Scaling of Water Governance Tasks: A Comparative Federal Analysis of the European Union and Australia ", Environmental Management, vol. $46, \mathrm{n}^{\circ} 1$, p. 7-16.

Betsill, M. et H. Bulkeley (2006). " Cities and the Multilevel Governance of Global Climate Change ", Global Governance, vol. 12, n² 2, p. 141-159.

Blanken, A. et G. Dewulf (2010). « PPPs in Health: Static or Dynamic? ", Australian Journal of Public Administration, vol. $69, \mathrm{n}^{\circ} 1$, p. 35-47.

Blum, J. (2008). "A Revisionist Model of Hospital Licensure ", Regulation \& Governance, vol. $2, n^{\circ} 1$, p. $48-64$.

Bradford, N. (2005). "Place-based Public Policy: Towards a New Urban and Community Agenda for Canada ", Canadian Policy Research Networks Research Report, nF/51.

Brown, G. R. (1994). « Canadian Federal-provincial Overlap and Presumed Government Inefficiency ", Publius, vol. 24, n 1, p. 21-37.

Bulkeley, H. (2006). « Urban Sustainability: Learning from Best Practice? ", Environment and Planning, vol. $38, \mathrm{n}^{\circ}$ 6, p. 1029-1044.

Bulkeley, H. et S. Moser (dir.) (2007). "Responding to Climate Change: Governance and Social Action beyond Kyoto ", Global Environmental Politics, vol. 7, n² 2, p. 1-144.

Contandriopoulos, D. et autres (2004). " Governance Structures and Political Processes in a Public System: Lessons from Quebec », Public Administration, vol. 8, n 3, p. 627-655.

Costanza, R. et J. Greer (1995). " The Chesapeake Bay and its Watershed: A Model for Sustainable Ecosystem Management? ", dans L. Gunderson, C. Holling et S. Light (dir.), Barriers and Bridges to the Renewal of Ecosystems and Institutions, New York, Columbia University Press, p. 169-213.

Dahlström, C., B. Peters et J. Pierre (2011). «Steering Strategies in Western Democracies », dans C. Dahlstom, B. Peters et J. Pierre (dir.), Steering from the Centre: Strengthening Political Control in Western Democracies, Toronto, University of Toronto Press, p. 263-276.

Durant, R. (2004). "Reconnecting with Stakeholders ", dans R. Durant, D. Fiorino et R. O'Leary (dir.), Environmental Governance Reconsidered: Challenges, Choices, and Opportunities, Cambridge, MIT Press, p. 177-182.

Durant, R., D. Fiorino et R. O'Leary (2004). «Introduction », dans R. Durant, D. Fiorino et R. O'Leary (dir.), Environmental Governance Reconsidered: Challenges, Choices, and Opportunities, Cambridge, MIT Press, p. 1-28.

Elson, S. (2005). The Evolution of Health System Governance in Canada and Ontario Including Cautionary Reflections on the Introduction of Local Health Integration Networks in Ontario, www.lhsc.on.ca/About_Us/ISAN/communication/articles/evolution.pdf (page consultée le 3 janvier 2010).

La gouvernance multiniveau et la nouvelle gestion publique : 
Gore, C. et P. Robinson (2009). " Local Government Response to Climate Change: Our Last, Best Hope? ", dans H. Selin et S. VanDeveer (dir.), Changing Climates in North American Politics: Institutions, Policymaking, and Multilevel Governance, Cambridge, MIT Press, p. 137-158.

Grodzins, M. (1960). The Report of the President's Commission on National Goals, The American Assembly Goals for Americans, Englewood Cliffs, Prentice Hall, réimprimé dans Grodzins, M. (2007), "The Federal System ", dans L. J. O’Toole (dir.), American Intergovernmental Relations: Foundations, Perspectives, and Issues Washington, DC, CQ Press, p. 54-63.

Gruening, G. (2001). " Origin and Theoretical Basis of New Public Management ", International Public Management Journal, vol. 4, $\mathrm{n}^{\circ}$ 1, p. 1-25.

Head, B. (2011). "Australian Experience: Civic Engagement as Symbol and Substance ", Public Administration and Development, vol. 31, n 2, p. 102-112.

Heimer, C. et autres (2008). " Health Care and New Governance: The Quest for Effective Regulation ", Regulation and Governance, vol. 2, n 1, p. 1-8.

Hoflund, A. et M. Farquhar (2008). "Challenges of Democratic Experimentalism: A Case Study of the National Quality Forum in Health Care », Regulation \& Governance, vol. $2, n^{\circ} 1$, p. 121-135.

Hollander, R. (2009). " Rethinking Overlap and Duplication: Federalism and Environmental Assessment in Australia ", Publius: The Journal of Federalism, vol. 40, $\mathrm{n}^{\circ} 1$, p. 136-170.

Hollander, R. et H. Patapan (2007). "Pragmatic Federalism: Australian Federalism from Hawke to Howard ", Australian Journal of Public Administration, vol. 66, n 3, p. 280-297.

Homburg, V., C. Pollitt et S. van Thiel (2007). «Introduction », dans V. Homburg, C. Pollitt et S. van Thiel (dir.), New Public Management in Europe: Adaptation and Alternatives, Houndmills, Palgrave, p. 1-9.

Hood, C. et G. Peters (2004). "The Middle Aging of New Public Management: Into the Age of Paradox? ", Journal of Public Administration Research and Theory, vol. 14, $\mathrm{n}^{\circ} 3$, p. 267-282.

Hooghe, L. et G. Marks (2003). "Unravelling the Central State, but How? Types of Multilevel Governance ", American Political Science Review, vol. 97, n² 2, p. 233-243.

Howlett, M. (2000). "Beyond Legalism: Policy Ideas, Implementation Styles and Emulation-based Convergence in Canadian and U.S. Environmental Policy ", Journal of Public Policy, vol. 20, n 3 , p. 305-329.

Huang, K. et K. Provan (2006). " Resource Tangibility and Patterns of Interaction in a Publicly Funded Health and Human Services Network ", Journal of Public Administration Research and Theory, vol. 17, p. 435-454.

Huque, A. et N. Watton (2010). "Federalism and the Implementation of Environmental Policy: Changing Trends in Canada and the United States ", Public Organization Review, vol. 10, $\mathrm{n}^{\circ}$ 1, p. 71-88.

John, D. (2004). " Civic Environmentalism ", dans R. Durant, D. Fiorino et R. O'Leary (dir.), Environmental Governance Reconsidered: Challenges, Choices, and Opportunities, Cambridge, MIT Press, p. 219-254.

Jones, S. (2009). "The Future of Renewable Energy in Australia: A Test for Cooperative Federalism? ", Australian Journal of Public Administration, vol. 68, n 1, p. 1-20. 
Jones, S. (2008). " Cooperative Federalism?: The Case of the Ministerial Council on Education, Employment, Training and Youth Affairs ", Australian Journal of Public Administration, vol. $67, \mathrm{n}^{\circ}$ 2, p. 161-172.

Karkkainen, B. (2004). " Post-Sovereign Environmental Governance », Global Environmental Politics, vol. 4, n 1, p. 72-96.

Kettl, D. (2009). "The Key to Networked Government ", dans S. Goldsmith et D. Kettl (dir.), Unlocking the Power of Networks: Keys to High-Performance Government, Washington, Brookings Institution Press, p. 1-14.

Kettl, D. (2005). The Global Public Management Revolution, Washington, Brookings Institution Press.

Kettl, D. (2000). " The Transformation of Governance: Globalization, Devolution, and the Role of Government ", Public Administration Review, vol. 60, n 6, p. 488-497.

Krantzberg, G. et E. Houghton (1996). "The Remedial Action Plan that Led to the Cleanup and Delisting of Collingwood Harbour as an Area of Concern ", Journal of Great Lakes Research, vol. 22, n 2 , p. 469-483.

Leo, C. et J. Enns (2009). " Multi-Level Governance and Ideological Rigidity: The Failure of Deep Federalism ", Canadian Journal of Political Science, vol. 42, n 1, p. 93-116.

Levy, R. (2010). "New Public Management: End of an Era? ", Public Policy and Administration, vol. 25, $\mathrm{n}^{\circ}$ 2, p. 234-240.

Lewis, C. et D. Marsh. (2012). " Network Governance and Public Participation in PolicyMaking: Federal Community Cabinets in Australia ", Australian Journal of Public Administration, vol. 71, $\mathrm{n}^{\circ} 1$, p. 6-19.

Marks, G., L. Hooghe et K. Blank (1996). " European Integration from the 1980s: StateCentric v. Multi-level Governance ", Journal of Common Market Studies, vol. 34, n 3, p. 341-378.

Newig, J. et O. Fritsch (2009). « Environmental Governance: Participatory, Multi-Level and Effective? ", Environmental Policy and Governance, vol. 19, n 3, p. 197-214.

Ongaro, E. et autres (2011). Policy, Performance and Management in Governance and Intergovernmental Relations: Transatlantic Perspectives, Cheltenham, Edward Elgar.

Ongaro, E. et autres (dir.) (2010). Governance and Intergovernmental Relations in the European Union and the United States: Theoretical Perspectives, Cheltenham, Edward Elgar.

Osborne, S. P. (2010). «Introduction », dans S. P. Osborne (dir.) The New Public Governance? Emerging Perspectives on the Theory and Practice of Public Governance, Abingdon, Routledge, p. 1-16.

Painter, M. (2001). "Multi-level Governance and the Emergence of Collaborative Federal Institutions in Australia ", Policy \& Politics, vol. 24, n 2, p. 137-150.

Papillon, M. (2008). " Canadian Federalism and the Emerging Mosaic of Aboriginal Multilevel Governance ", dans H. Bakvis et G. Skogstad (dir.) Canadian Federalism: Performance, Effectiveness, and Legitimacy, Toronto, Oxford University Press, p. 291-313.

Peters, B. (2008). Governance Through the Political System: Making and Implementing Policy, http://paperroom.ipsa.org/papers/paper_4037.pdf (page consultée le 30 juillet 2012).

Peters, B. et J. Pierre (1998). " Governance without Government? Rethinking Public Administration ", Journal of Public Administration Research and Theory, vol. 8, n ${ }^{\circ} 2$, p. 223-243.

La gouvernance multiniveau et la nouvelle gestion publique : 
Piattoni, S. (2010). The Theory of Multi-level Governance: Conceptual, Empirical, and Normative Challenges, Oxford, Oxford University Press.

Piattoni, S. (2009). " Multi-level Governance: A Historical and Conceptual Analysis", Journal of European Integration, vol. 31, n 2, p. 163-180.

Pollitt, C. et G. Bouckaert (2011). Public Management Reform: A Comparative Analysis: New Public Management, Governance, and the Neo-Weberian State, $3^{e}$ éd., Oxford, Oxford University Press.

Posner, P. (2009). "Networks in the Shadow of Government: The Chesapeake Bay Program ", dans S. Goldsmith et D. Kettl (dir.) Unlocking the Power of Networks: Keys to High-performance Government, Washington, Brookings Institution Press, p. 62-94.

Prager, K. (2010). « Local and Regional Partnerships in Natural Resource Management: The Challenge of Bridging Institutional Levels ", Environmental Management, vol. 46, p. 711-724.

Provan, K., K. Huang et H. Milward (2009). « The Evolution of Structural Embeddedness and Organizational Social Outcomes in a Centrally Governed Health and Human Services Network ", Journal of Public Administration Research and Theory, vol. 19, p. 873893.

Rabe, B. (2009a). " Governing the Climate from Sacramento ", dans S. Goldsmith et D. Kettl (dir.), Unlocking the Power of Networks: Keys to High-performance Government, Washington, Brookings Institution Press, p. 34-61.

Rabe, B. (2009b). "Second-Generation Climate Policies in the States Proliferation, Diffusion, and Regionalization ", dans H. Selin et S. VanDeveer (dir.), Changing Climates in North American Politics: Institutions, Policymaking, and Multilevel Governance, Cambridge, MIT Press, p. 67-86.

Rabe, B. (2007). " Beyond Kyoto: Climate Change Policy in Multilevel Governance Systems ", Governance, vol. 20, n 3, p. 423-444.

Rhodes, R. (2000). " Governance and Public Administration ", dans J. Pierre (dir.), Debating Governance: Authority, Steering, and Democracy, Oxford, Oxford University Press, p. 54-90.

Sabel, C., A. Fung et B. Karkkainen (2000). "Beyond Backyard Environmentalism ", dans J. Cohen et J. Rogers (dir.), Beyond Backyard Environmentalism, Cambridge, Beacon Press.

Scheberle, D. (2004a) Federalism and Environmental Policy: Trust and the Politics of Implementation, Washington, Georgetown University Press.

Scheberle, D. (2004b). "Devolution ", dans R. Durant, D. Fiorino et R. O'Leary (dir.), Environmental Governance Reconsidered: Challenges, Choices, and Opportunities, Cambridge, MIT Press, p. 361-392.

Schlager, E. (2004). " Common Pool Resource Theory ", dans R. Durant, D. Fiorino et R. O'Leary (dir.), Environmental Governance Reconsidered: Challenges, Choices, and Opportunities, Cambridge, MIT Press, p. 145-176.

Schön-Quinlivan, E. (2011). Reforming the European Commission, New York, Palgrave Macmillan.

Selin, H. et S. VanDeveer (dir.) (2009a). Changing Climates in North American Politics: Institutions, Policymaking, and Multilevel Governance, Cambridge, MIT Press. 
Selin, H. et S. VanDeveer (2009b). " Changing Climates and Institution Building across the Continent ", dans H. Selin et S. VanDeveer (dir.), Changing Climates in North American Politics: Institutions, Policymaking, and Multilevel Governance, Cambridge, MIT Press, p. 3-22.

Selin, H. et S. VanDeveer (2009c). « North American Climate Governance Policymaking and Institutions in the Multilevel Greenhouse ", dans H. Selin et S. VanDeveer (dir.), Changing Climates in North American Politics: Institutions, Policymaking, and Multilevel Governance, Cambridge, MIT Press, p. 305-326.

Smith, R. et J. Lavoie (2008). « First Nations Health Networks: A Collaborative System Approach to Health Transfer ", Healthcare Policy, vol. 4, n² 2, p. 101-112.

Stein, M. et L. Turkewitsch (2011a). "The Relevance of the Concept of Multilevel Governance for a Comparative Analysis of Canadian and American Intergovernmental Relations ", dans H. Enderlein, S. Wälti et M. Zürn (dir.), Handbook on Multi-level Governance, Cheltenham, Edward Elgar, p. 184-202.

Stein, M. et L. Turkewitsch (2011b). " An Assessment of Multi-level Governance as an Analytical Concept Applied to Federations and Decentralized Unitary Systems: Germany versus the United Kingdom ", Communication présentée au symposium European Consortium for Political Research (ECPR) 6th General Conference, University of Iceland, Reykjavik, 25-27 août.

Stein, M. et L. Turkewitsch (2010a). «The European Model of Governance Beyond Europe: The Applicability of the Concept of Multi-Level Governance to the Comparative Study of Intergovernmental Relations in Parliamentary and Presidential Federations ", Communication présentée au symposium IPSA International Conference "Is There a European Model of Governance? A Comparative Perspective", Luxembourg, 18-20 mars.

Stein, M. et L. Turkewitsch (2010b). " Multilevel Governance and Federalism: Closely Linked or Incompatible Concepts? ", Participation, vol. 34, n 2, p. 3-5.

Stein, M. et L. Turkewitsch (2009). " Similarities and Differences in Patterns of Intergovernmental Relations in Parliamentary and Presidential Federations: A Comparative Analysis ", Communication présentée au symposium International Political Science Association (IPSA) 21st World Congress of Political Science, Santiago, 14 juillet.

Stein, M. et L. Turkewitsch (2008a). « The Concept of Multi-level Governance in Studies of Federalism ", Communication présentée au symposium International Political Science Association (IPSA) International Conference, Université Concordia, Montréal, 2 mai.

Stein, M. et L. Turkewitsch (2008b). "The Concepts of Federalism and Multi-level Governance Evaluated: A Comparative Analysis of Canadian and American Intergovernmental Relations ", Communication présentée au symposium The International Political Science Association's Research Committee 28 - Comparative Federalism and Federation Annual Conference, Berlin, Hertie School of Governance, 3-4 octobre.

Trubek, L. (2006). " New Governance and Soft Law in Health Care Reform ", Indiana Health Law Review, vol. 3, p. 139-169.

Trubek, L. et D. Trubek (2005). « Hard and Soft Law in the Construction of Social Europe: the Role of the Open Method of Co-ordination ", European Law Journal, vol. 11, n 3 , p. 343-364. 
Valiante, M. (2002). « Legal Foundations of Canadian Environmental Policy: Underlining our Values in a Shifting Landscape ", dans D. VanNijnatten et R. Boardman (dir.), Canadian Environmental Policy: Context and Cases, Toronto, Oxford University Press, p. 5-24.

Van Kersbergen, K. et F. Van Waarden (2004). " "Governance” as a Bridge between Disciplines: Cross-Disciplinary Inspiration Regarding Shifts in Governance and Problems of Governability, Accountability and Legitimacy ", European Journal of Political Research, vol. 43, n² 2, p. 143-171.

Vining, A. et D. Weimer (2005). "Economic Perspectives on Public Organizations ", dans E. Ferlie, L. Lynn et C. Pollitt (dir.), The Oxford Handbook of Public Management, Oxford, Oxford University Press, p. 209-233.

Young, R. et C. Leuprecht (2004). «Introduction: New Work, Background Themes, and Future Research about Municipal-Federal-Provincial Relations in Canada ", dans R. Young et C. Leuprecht (dir.), Canada: The State of the Federation 2004: MunicipalFederal-Provincial Relations in Canada, Montréal et Kingston, McGill-Queen’s University Press, p. 3-24.

Zurba, M. et autres (2012). " Building Co-Management as a Process: Problem Solving Through Partnerships in Aboriginal Country, Australia ", Environmental Management, vol. 49, n 6, p. 1130-1142. 